\title{
Protecting the tuberculosis drug pipeline: stating the case for the rational use of fluoroquinolones
}

\author{
Giovanni Battista Migliori*,\#\#, Miranda W. Langendam*,\#\#, Lia D’Ambrosio*, \\ Rosella Centis*, Francesco Blasi ${ }^{\mp,}$ Emma Huitric ${ }^{+}$, Davide Manissero ${ }^{+}$ \\ and Marieke J. van der Werf ${ }^{\S, f}$
}

ABSTRACT: The use of fluoroquinolones (FQs) to treat lower respiratory tract infections (LTRI) other than tuberculosis (TB) allows selection of FQ-resistant TB when TB is misdiagnosed. This study maps national guidelines on the use of FQs for LRTI in Europe and determines the risk of FQ-resistant TB upon FQ treatment before TB diagnosis.

A questionnaire was developed to map existing national LRTI and community-acquired pneumonia (CAP) guidelines. A systematic review and meta-analysis were performed to determine the risk of FQ-resistant TB if prescribed FQs prior to TB diagnosis.

$15(80 \%)$ out of 24 responding European Respiratory Society national delegates reported having national LRTI management guidelines, seven including recommendations on FQ use and one recommending $F Q s$ as the first-choice drug. 18 out of 24 countries had national CAP management guidelines, two recommending $F Q$ as the drug of choice. Six studies investigating $F Q$ exposure and the risk of FQ-resistant TB were analysed. TB patients had a three-fold higher risk of having FQ-resistant TB when prescribed FQs before TB diagnosis, compared to non FQexposed patients (OR 2.81, 95\% Cl 1.47-5.39).

Although the majority of European countries hold national LRTI/CAP guidelines, our results suggest that a risk of developing $F Q$ resistance exists. Further strengthening of, and adherence to, guidelines is needed to ensure rational use of FQs.

KEYWORDS: Drug resistance, fluoroquinolones, lower respiratory tract infections, tuberculosis

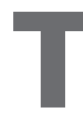

he rational use of antibiotics has recently attracted major attention, being selected as the topic of the 2011 World Health Day [1-3]. Drug resistant tuberculosis (TB) is a growing threat to the control and ultimate elimination of TB [4-7]. Not only has multidrug-resistant (MDR)-TB $[8,9]$ established itself within the European Union (EU) borders, but extensively drug-resistant (XDR)-TB $[10,11]$ has also become an issue. More recently, debate was initiated to define as extremely drugresistant (XXDR)-TB or total drug resistant (TDR)TB, TB cases that harbour Mycobacterium tuberculosis strains with resistance to all the known drugs [12-14]. The World Health Organization (WHO) has initiated discussions on the eventual need to develop a definition for such resistant TB strains. At present, however, an international, expert-endorsed definition has not yet been agreed.

Drug resistance in $M$. tuberculosis occurs through spontaneous chromosomal mutations $[15,16]$ and, depending on the drug target, resistance to a specific drug occurs at a specific rate (i.e. every $\mathrm{x}$ number of bacilli will have a mutation conferring resistance to a specific drug). This in itself forms the basis and rationale for multi-drug TB regimens, targeting the bacilli from several angles to ensure all bacilli are killed.

Recent research has shown that the use of fluoroquinolones (FQs), one of the key second-line drugs for the treatment of MDR-TB [17-22] also used to treat other lower respiratory tract infections

\section{AFFILIATIONS}

*WHO Collaborating Centre for TB and Lung Diseases, Fondazione S.

Maugeri, Care and Research Institute, Tradate,

'Respiratory Medicine Section, Dipartimento Toraco-Polmonare e Cardiocircolatorio, University of Milan, IRCCS Fondazione Cà Granda, Milan, Italy.

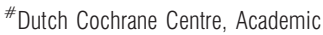
Medical Center, University of Amsterdam,

${ }^{\mathrm{s}}$ Center for Infection and Immunity Amsterdam (CINIMA), Academic Medical Center, University of Amsterdam, Amsterdam, ${ }^{f}$ KNCV Tuberculosis Foundation, The Hague, The Netherlands.

+European Centre for Disease Prevention and Control (ECDC), Stockholm, Sweden.

\#\#These authors contributed equally.

CORRESPONDENCE

E. Huitric

European Centre for Disease

Prevention and Control (ECDC)

Tomtebodavägen 11a

17183 Stockholm

Sweden

E-mail: emma.huitric@

ecdc.europa.eu

Received:

March 012012

Accepted after revision:

April 032012

First published online:

May 312012

This article was modified in April 2016 to correct errors in the licence information.

European Respiratory Journal Print ISSN 0903-1936

Online ISSN 1399-3003 
(LRTIs), poses a potential risk for selecting FQ-resistant strains of M. tuberculosis in case TB disease is misdiagnosed.

The EU is a heterogeneous setting with regard to TB incidence, with several member states having a low-incidence of the disease $[23,24]$. This in itself presents the possibility that TB diagnosis is not considered when patients present with a persistent cough, and that patients may be erroneously treated for another LRTI. Being a broad-spectrum antibiotic effective against several respiratory infections, FQs can be a choice of treatment for such infections. There is, in other words, the risk of selecting FQ-resistant strains (regardless of resistance pattern to other drugs) if FQs are used in LRTI without the prior exclusion of TB disease.

In this manuscript, we aimed to: 1) assess recommendations on FQ use for LRTIs other than TB by mapping existing national guidelines for the treatment of LRTI/community-acquired pneumonia (CAP) in EU/European Economic Area (EEA) member states; and 2) assess whether treatment with FQs before the diagnosis of TB is associated with a higher risk of FQ-resistant TB.

\section{DEFINITIONS}

World Health Organization (WHO): the United Nations agency directing and coordinating public health priorities.

European Centre for Disease Prevention and Control (ECDC): EU agency mandated to identify, assess and communicate current and emerging threats to human health posed by infectious diseases. The ECDC is located in Stockholm, Sweden.

European Respiratory Society (ERS): one of the leading scientific societies focused on respiratory medicine.

TB: defined as the clinical, bacteriological and/or radiographical manifest disease $[6,18,25]$.

MDR-TB: TB caused by $M$. tuberculosis strains resistant to at least the two first-line anti-TB drugs, isoniazid (INH) and rifampicin (RMP) [11].

XDR-TB: TB caused by M. tuberculosis resistant to RMP and INH (i.e. MDR-TB) plus any FQ, and at least one of the three following injectable drugs: capreomycin, kanamycin or amikacin [11].

XXDR-TB: M. tuberculosis strains resistant to all available firstand second-line drugs. Of note, there is currently no internationally endorsed definition of XXDR-TB. A definition of TB cases resistant to all TB drugs is currently being discussed and $\mathrm{WHO}$ is in the process of assessing the need to develop an official definition [12-14].

TDR-TB: M. tuberculosis strains resistant to all first- and secondline drugs tested. Of note, there is currently no internationally endorsed definition of TDR-TB, this is a definition proposed by authors based on a recent publication [12]. A definition of TB cases resistant to all TB drugs is currently being discussed and $\mathrm{WHO}$ is in the process of assessing the need to develop an official definition $[12,26]$.

Low TB incidence country: countries with a crude notification rate $<20$ per 100,000 population $[7,24]$.

LRTI: an acute illness (present for $\leqslant 21$ days) usually with cough as the main symptom, with at least one other lower respiratory tract symptom (sputum production, dyspnoea, wheeze or chest discomfort/pain) and no alternative explanation (e.g. sinusitis or asthma).

Suspected CAP: an acute illness with cough and at least one of new focal chest signs, fever for $>4$ days or dyspnoea/ tachypnoea, and without other obvious cause [21, 22].

Definite CAP: as for suspected CAP, but supported by chest radiograph findings of lung shadowing that is likely to be new. In the elderly, the presence of shadowing on a chest radiograph accompanied by acute clinical illness (unspecified) without other obvious cause.

TB control: strategies aimed to reduce the incidence of new infections with $M$. tuberculosis complex by identifying sources of infection as rapidly as possible and rendering them noninfectious through curative treatment. Currently, non-infectious cases are also a priority for TB control to reduce human suffering, including in children [27].

TB elimination: the point at which less than one case per $1,000,000$ inhabitants emerges annually in the general population $[7,24,28]$.

Practical Approach to Lung Health (PAL): this is a WHOlaunched programme aimed at integrating the clinical and public health approach to respiratory symptoms and diseases [29].

\section{METHODS}

\section{Assessing recommendations on the use of $F Q$ in the $E U$ for LRTI other than TB by mapping existing national guidelines for the treatment of LRTI/CAP}

A questionnaire was developed by the ECDC in collaboration with the ERS and aimed at: 1) mapping the existing national guidelines for the management of LTRI and CAP; 2) assessing the extent to which FQs are recommended for the treatment of LRTIs other than TB and availability of information on the risk of TB patients developing FQ resistance if misdiagnosed with another LRTI and treated with FQs; and 3) assessing whether the subsequent recommendations on the use of FQs for treatment included the need to exclude TB prior to treatment.

The questionnaire was divided into two sections and consisted of 10 questions with a yes/no answer investigating the guidelines on LRTI and CAP (table 1). The questionnaire was sent by the ERS (Lausanne, Switzerland) to 30 ERS national delegates belonging to the EU/EEA member states. A reminder was sent to the late respondents 15 days after the first invitation. In case of no response, the questionnaires from these countries were received by collaboration of the ERS officers in these countries, in order to achieve a satisfactory response rate (80\%).

\section{Estimating the risk for FQ-resistant TB if treated with FQ prior to TB diagnosis}

A systematic review to assess the association between the use of FQs for CAP and other respiratory infections and FQ-resistant $\mathrm{TB}$ had been recently performed by CHEN et al. [30]. We assessed the quality of the systematic review with the AMSTAR checklist [31] and concluded that the review by CHEN et al. [30] was well performed. However, the risk of bias of the included studies was not assessed by the authors [30]. We decided to update the results and to assess the risk of bias of the included studies. 
TABLE 1 Questionnaire on lower respiratory tract infections (LRTIS) and community-acquired pneumonia (CAP) guidelines sent to 30 European Respiratory Society (ERS) delegates of European Union/European Economic Area countries

\section{Guidelines for the management of LRTIs}

1. Does your country have national/sub-national guidelines for the management of LRTIs?

2. Do the guidelines for the management of LRTIs consider the ERS guidelines for the management of adult respiratory tract infections?

3. Do the guidelines for the management of LRTIs include recommendations on the differential diagnosis, treatment and management of TB?

a. If no, do the national guidelines for the management of LRTIs include information on how and where to refer suspected TB patients for diagnosis, treatment and management?

4. Do the guidelines for the management of LRTIs include recommendations on the use of FQ in LRTIs and the risk for development of FQ-resistant TB in misdiagnosed patients?

5. Do the guidelines for the management of LRTI recommend FQ as:

a. First drug of choice to treat LRTI?

b. Second drug of choice to treat LRTI?

II. Guidelines for the management of CAP

1. Does your country have national/sub-national guidelines specifically for the management of CAP?

2. Do the guidelines for the management of CAP consider the ERS guidelines for the management of adult respiratory tract infections?

3. Do the guidelines for the management of CAP include recommendations on the differential diagnosis and treatment of TB?

4. If no, do the national guidelines for the management of CAP include information on how and where to refer suspected TB patients for diagnosis, treatment and management?

5. Do the guidelines for the management of CAP include recommendations on the use of FQ in CAP and the risk of development of FQ-resistant TB in misdiagnosed patients?

6. Do the guidelines for the management of CAP recommend FQ as:

a. First drug of choice to treat CAP?

b. Second drug of choice to treat CAP?

TB: tuberculosis; FQ: fluoroquinolones.

For the update, we searched the literature (January 1, 2010 to January 18, 2011) for relevant studies. Compared with CHEN et al. [30], our search strategy included more keywords and $\mathrm{MeSH}$ terms (Annex 1 in supplementary material). We did not search CINAHL [32] as this database was unlikely to provide any relevant papers. We did search the TRIP database [33]. Ongoing randomised control trials on FQs (which might report on adverse effects) were searched via the WHO International Clinical Trials Registry Platform [34].

For the updated search, one investigator selected studies for eligibility. A $10 \%$ random sample was assessed by a second investigator and compared with the assessment of the first investigator. If there had been relevant inconsistencies all articles would have been evaluated by the second author. One investigator extracted all relevant data items from the included studies. A second investigator independently extracted the main results of the included studies and checked the extract of a subsample of the articles. Consensus was reached by discussion. The meta-analysis was performed as stated by CHEN et al. [30]. Two investigators independently assessed the risk of bias of the included studies using the Newcastle Ottawa Scale (NOS) for cohort studies [35].

\section{RESULTS}

Assessing recommendations on the use of $F Q$ in the $E U$ for LRTI other than TB by mapping national guidelines for the treatment of LRTI/CAP

$24(80 \%)$ out of 30 national delegates from the following countries responded to the questionnaire: Austria, Belgium, Bulgaria, Czech Republic, Denmark, Estonia, Finland, France, Germany,
Greece, Ireland, Italy, Latvia, Lithuania, Luxembourg, Malta, the Netherlands, Norway, Poland, Portugal, Romania, Slovakia, Spain, UK.

At the time of the survey, 15 (62\%) of the 24 responding national delegates had national/sub-national guidelines for the management of LRTIs, of which seven $(47 \%)$ included recommendations on the differential diagnosis, treatment and management of TB and seven the recommendation on the use of FQs in LTRI and the risk of FQ-resistant TB in misdiagnosed patients. Only one of the 15 guidelines recommended FQs as the first drug of choice to treat LRTIs and $10(67 \%)$ recommended their use as a second drug of choice.

$18(75 \%)$ out of the 24 responding national delegates reported having national/sub-national guidelines specifically for the management of CAP at the time of the survey, of which, seven (39\%) included recommendations on the differential diagnosis, treatment and management of TB and seven the recommendation on the use of FQs in CAP and the risk of FQ-resistant TB in misdiagnosed patients. Only two of the 18 guidelines recommended FQs as the first drug of choice to treat CAP and $12(67 \%)$ recommended their use as a second drug of choice. The detailed results are summarised in table 2 .

\section{Estimating the risk for FQ-resistant $T B$ if treated with $F Q$ prior to TB diagnosis}

Search results in the update

The update of the search identified 192 records; one study was eligible for inclusion [36]. In total, up to January 18, 2011, six studies investigating FQ exposure and the risk of FQ-resistant TB were included [36-41]. The most recently published study 
TABLE 2 Summary of the answers to the survey on lower respiratory tract infections (LRTI) and community-acquired pneumonia (CAP) guidelines by European Respiratory Society (ERS) delegates of 24 European Union/European Economic Area countries

\section{Guidelines for the management of LRTIs}

1. $15(62 \%)$ out of 24 countries have national/sub-national guidelines

2. $10(67 \%)$ out of 15 consider the ERS guidelines for the management of adult respiratory tract infections

3. 7 (47\%) out of 15 include recommendations on the differential diagnosis, treatment and management of TB while 8 (53\%) out of 15 do not include these recommendations and only 2 of these (25\%) include information on how and where to refer suspected TB patients for diagnosis, treatment and management

4. 7 (47\%) out of 15 include recommendations on the use of FQ in LRTI and the risk of developing FQ-resistant TB in misdiagnosed patients

5. 1 (7\%) out of 15 recommend FQ as first drug of choice to treat LRTI, 10 (67\%) out of 15 recommend FQ as second drug of choice to treat LRTI

\section{Guidelines for the management of CAP}

1. $18(75 \%)$ out of 24 countries have national/sub-national guidelines

2. $11(61 \%)$ out of 18 consider the ERS guidelines for the management of adult respiratory tract infections

3. 7 (39\%) out of 18 include recommendations on the differential diagnosis and treatment of TB while 11 (61\%) out of 18 do not include these recommendations and

only 3 of these (27\%) include information on how and where to refer suspected TB patients for diagnosis, treatment and management

4. $8(44 \%)$ out of 18 include recommendations on the use of FQ in CAP and the risk of developing FQ-resistant TB in misdiagnosed patients

5. $2(11 \%)$ out of 18 recommend FQ as first drug of choice to treat CAP, 12 (67\%) out of 18 recommend FQ as second drug of choice to treat CAP

TB: tuberculosis; FQ: fluoroquinolones.

[36] provided an overview of all relevant studies, and these include the same studies as in the review by CHEN et al [30].

\section{Description of the included studies}

Table 3 summarises the characteristics of the included studies. The exposure period, i.e. the period in which FQ exposure (through prescription) was measured, varied between 100 days and 12 months before TB diagnosis. One study did not report this period [39]. The proportion of TB cases exposed to FQs prior to TB diagnosis varied between $1.4 \%$ and $35 \%$.

\section{Risk of bias assessment}

The NOS star template in table 4 presents the results of the risk of bias assessment. It cannot be ruled out that, for all studies, the outcome of interest (e.g. FQ resistance) was already present at the time of exposure. For FQ-exposed patients this might be the case, as it is possible that FQ were prescribed for symptoms actually due to TB sustained by $M$. tuberculosis strains already resistant to FQ (but wrongly assumed to be caused by CAP). This may result in an overestimation of the association between FQ-exposure and FQ-resistant TB.

Three studies were adjusted for confounders [36, 40, 41]. Although the other included studies did not adjust for confounders, no baseline differences likely to be associated to FQ resistance were found and comparability was considered possible.

Other potential sources of bias we assessed were representativeness [36], selective inclusion [36, 37] and incomplete outcome assessment [36, 38, 41]. In all the studies analysed, quality was scored as moderate to high. As all studies are retrospective, data on FQ use may have been underestimated. However, this misclassification is likely to be similar for FQresistant and FQ-susceptible TB patients.

\section{Data synthesis}

Figure 1 presents the updated meta-analysis results. TB patients had a 2.81 (95\% CI 1.47-5.39) higher risk of FQ-resistant TB when they had been prescribed FQs before TB diagnosis than TB patients not exposed to FQs.

\section{DISCUSSION}

Recently, 10 compounds have progressed to the clinical development pipeline for the treatment of TB; two of which belong to the FQs (fig. 2) [42]. Other FQs are already available for the treatment of TB (e.g. ofloxacin). These new compounds, if properly managed, have the potential to become part of a future regimen that could positively affect the global TB control effort. There are two main threats that may result in the development of resistance to new compounds: 1) their use within inappropriate $\mathrm{TB}$ regimens; and 2) their use for $\mathrm{CAP}$ and other respiratory infections, and the subsequent risk for the emergence of resistant $\mathrm{TB}$, this regards especially the $\mathrm{FQ}$.

The first aim of this review was to assess existing recommendations on the use of FQs in the EU for LRTIs other than TB by mapping existing national guidelines for the treatment of LRTI/ CAP. At the time of the survey, not all national guidelines on LTRI and/or CAP highlighted the need to consider differential diagnosis of TB. Furthermore, among existing LRTI and CAP guidelines only seven informed on the potential risk of developing FQ resistance in misdiagnosed TB patients. In the EU setting, composed of numerous low-incidence countries where medical doctors have little opportunity to investigate a TB patient, there is a real risk that TB is not considered when a coughing patient reports to healthcare services. Maintaining the "know-how" on TB, e.g. ensuring that TB patients are correctly diagnosed, is essential to stop disease transmission in the population and further prevent drug resistance development $[18,24,25,29]$. Accessing clinicians through guidelines is a key channel to maintain know-how and keep TB high on the clinical agenda. Updating national and sub-national guidelines for the treatment of LRTI and CAP is therefore warranted, as well as describing the need for TB differential diagnosis and the risks behind FQs.

The recommendations included in national guidelines should be in line with PAL [29] and the International Standards for TB Care [25]. The recently launched EU Standards for TB Care [18] offer a further source to tailor TB standards to the EU. A 


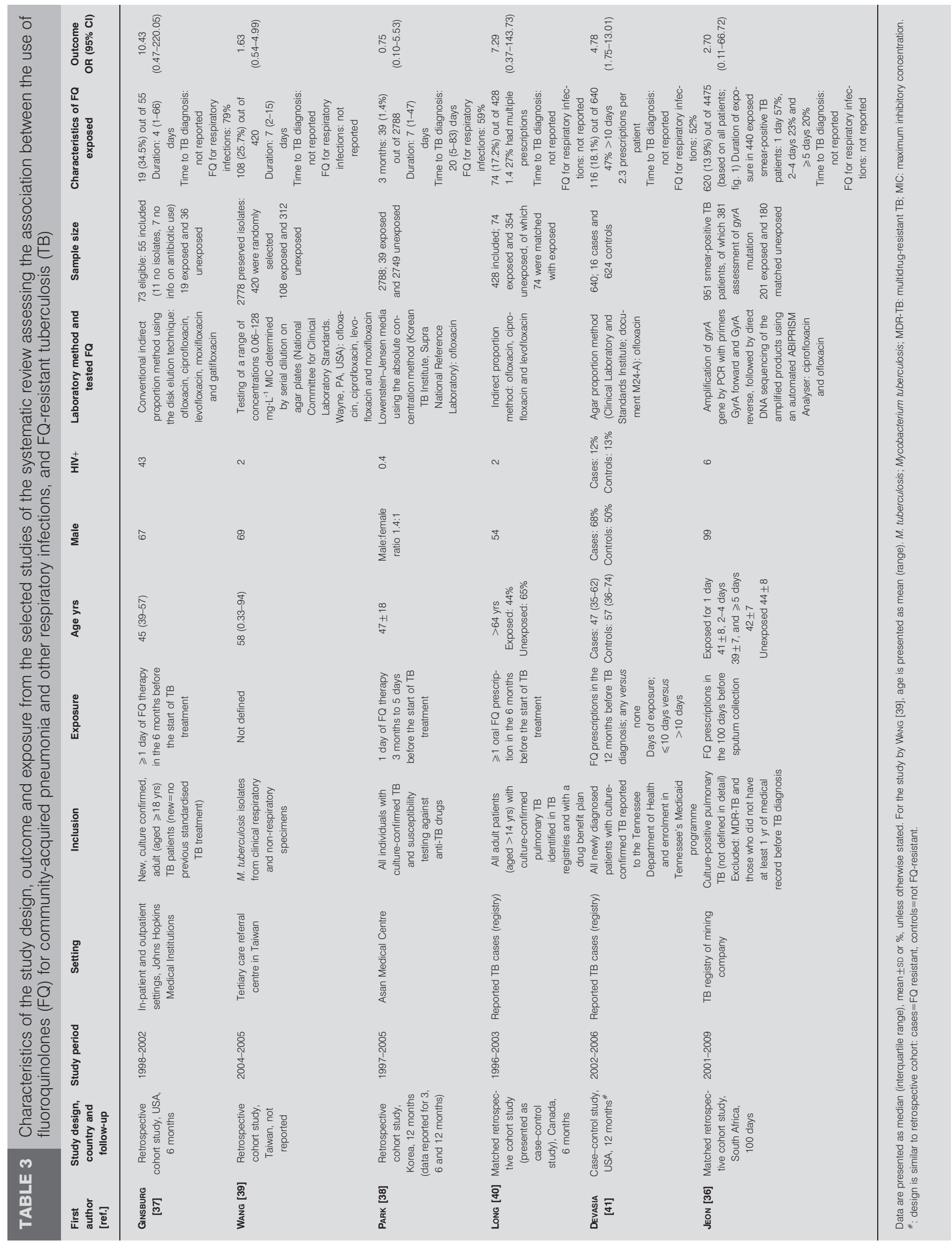




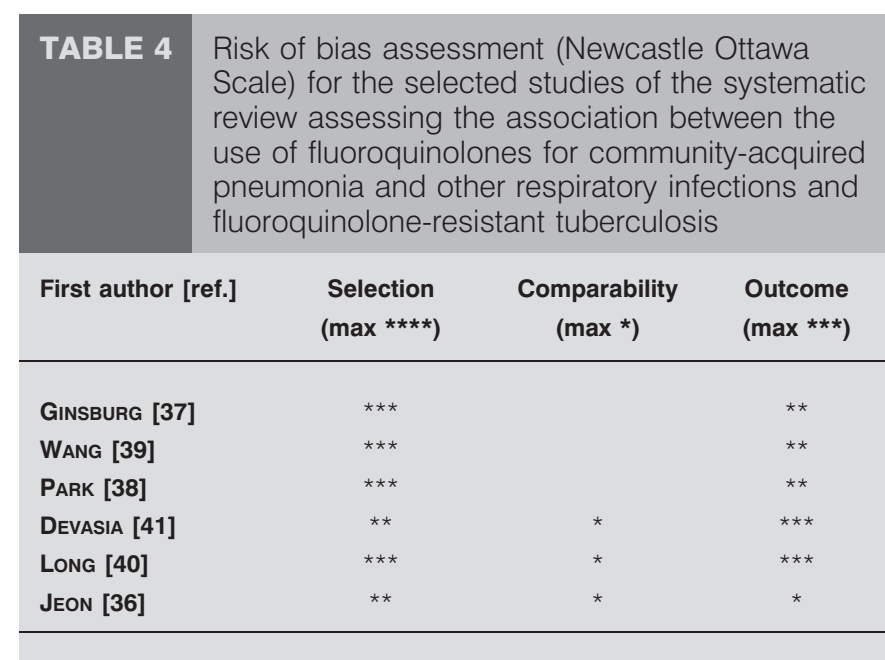

reassuring finding was that 10 LRTI guidelines and 12 CAP guidelines only recommended FQs as the second drug of choice for treatment, indicating the prudent use of this broadspectrum antibiotic.

The second aim of this review was to assess whether treatment with FQs before TB diagnosis is associated with a higher risk of FQ-resistant TB. All studies started by identifying patients with culture-confirmed $\mathrm{TB}$ and measured FQ resistance in these patients. FQ exposure was assessed by linkage with medical records or pharmacy registers.

In total, six studies of moderate-to-high quality were included. TB patients had a three-fold higher risk of FQ-resistant TB when they had been prescribed FQs before TB diagnosis, compared to TB patients who were not exposed to FQs (OR 2.81, 95\% CI 1.47-5.39). This is comparable to the OR of 2.7 reported by CHEN et al. [30].

The presence of FQ resistance at the time of FQ exposure would give an overestimation of the association between FQ exposure and FQ resistance. The risk of bias is high if pre-existing FQ resistance is related to exposure status. In this review we found some indirect evidence for this hypothesis: four out of the six studies included re-treatment cases, which is associated with drug resistance [44]. Moreover, the study of JEON et al. [36] showed a higher percentage of re-treatment cases among the FQexposed patients (table 2). WANG et al. [39] found a higher level of FQ resistance among the re-treatment cases: $7.9 \%$ versus $2.5 \%$.

The aim was to investigate FQ prescription for CAP or other respiratory infections. However, FQ were prescribed for other indications as well. It seems unlikely that this has an effect on the mechanism of development of FQ resistance. However, if FQs are prescribed for indications other than CAP it is less likely that the symptoms for which it was described were actually due to undiagnosed pulmonary TB. In these cases, monotherapy with FQs cannot be considered inappropriate and intensifying the diagnostic process by excluding TB before prescribing FQs does not seem relevant.

A recently published study by ADRIAENSSENS et al. [45] reports on the European outpatient use of FQs and offers several elements for a fruitful integration with the data generated by our study. Data on more than a decade of outpatient quinolone use were collected within the European Surveillance of Antimicrobial Consumption project, funded by ECDC [45].

Earlier FQs (including ofloxacin, levofloxacin and, particularly, ciprofloxacin) were seen to be the most frequently used in the 33 European countries surveyed between 1997 and 2009. Among the newer FQs, moxifloxacin and, to a lesser extent, prulifloxacin, were widely prescribed between 1997 and 2009, although a significant reduction in prescription was then observed starting from 2005 to 2006 in Germany, France, Hungary, Spain, Italy and the Netherlands. The survey showed that the prescription of FQs was highest in Southern Europe, intermediate in Eastern Europe and lowest in Northern Europe, with winter-related prescription waves correlating with the overall magnitude of FQ consumption.

The authors conclude that the overall increase in FQ prescription reflects the shift from the earlier FQs (aimed at treating urinary tract infections) to the newer, more recent "respiratory"

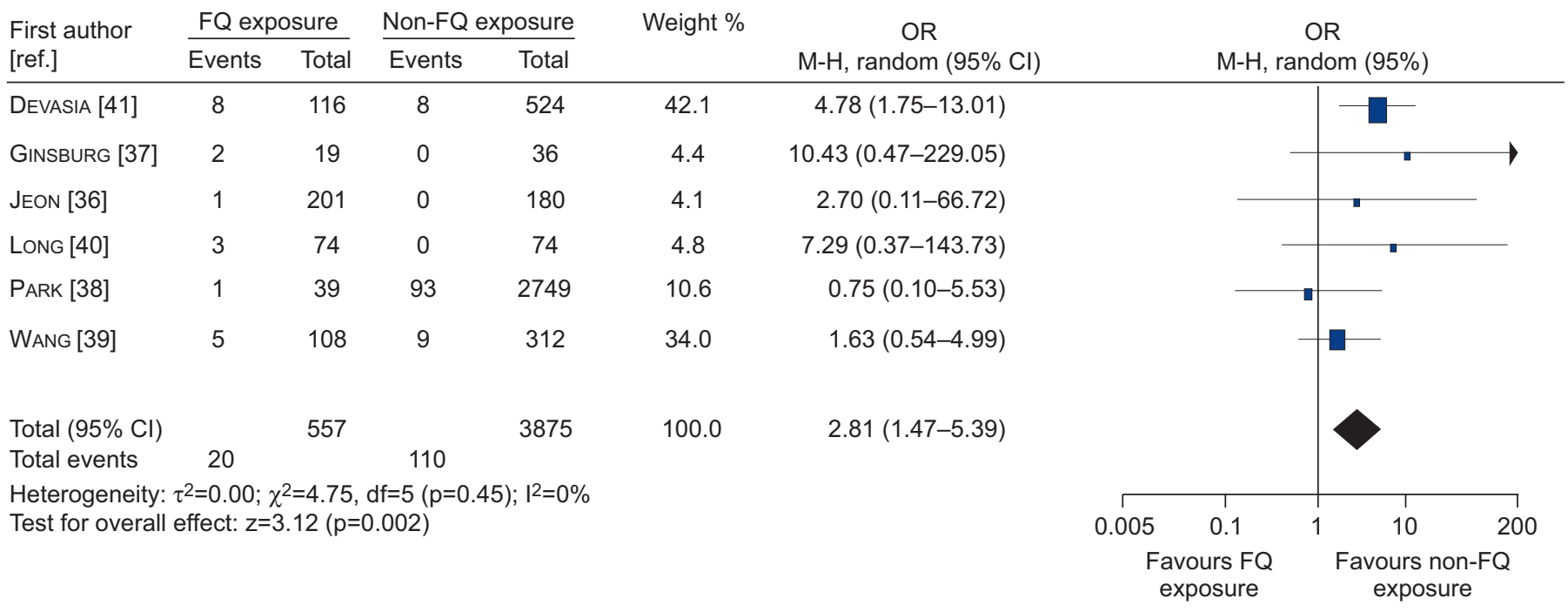

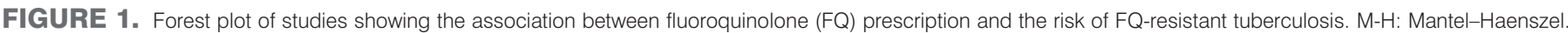




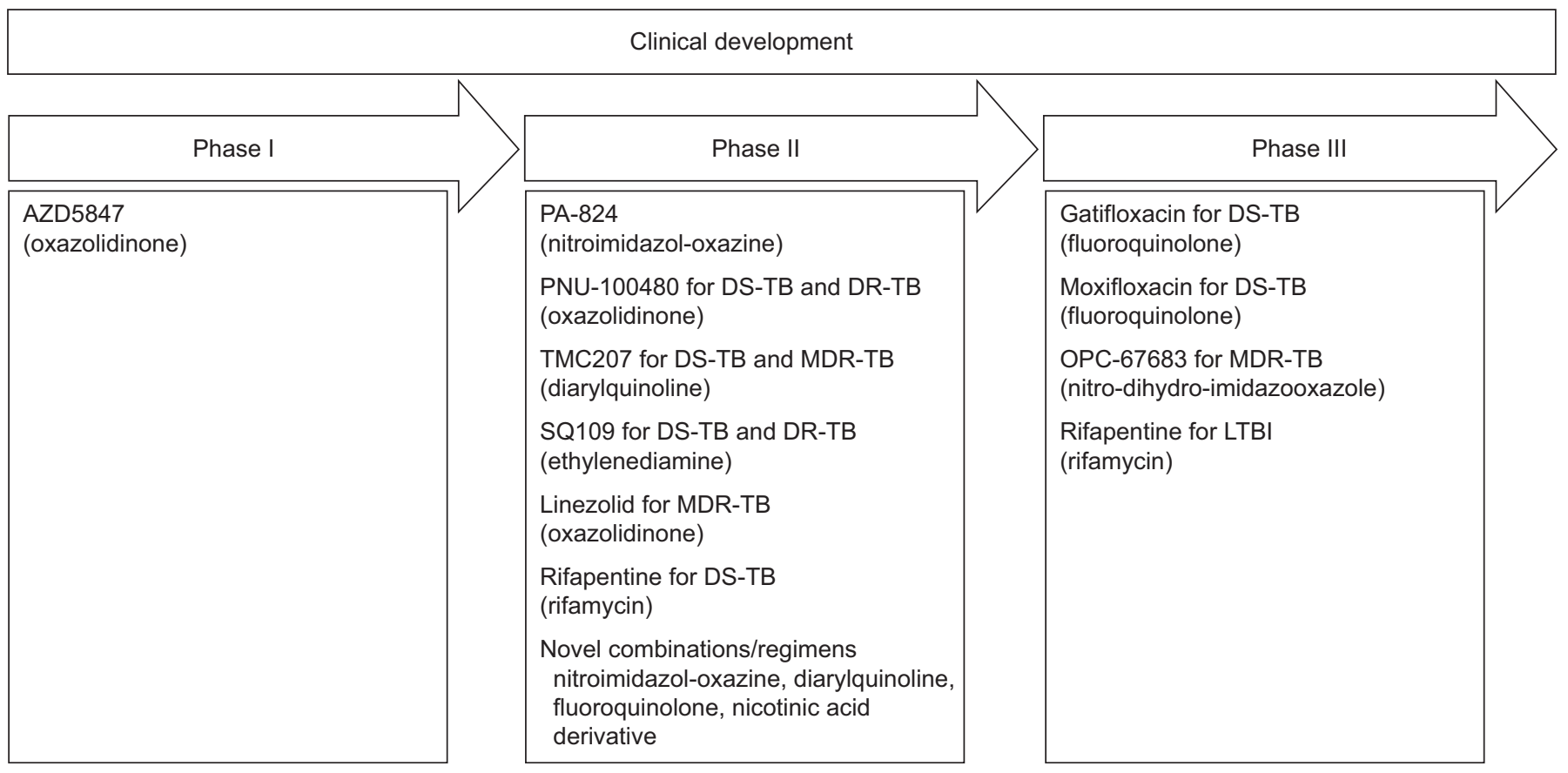

FIGURE 2. New drugs in the clinical development pipeline. 10 compounds are currently under clinical development for the treatment of drug-susceptible (DS), drugresistant (DR) or multi-drug resistant (MDR) tuberculosis (TB). Each compound name, group and position in the clinical development pipeline is listed. Gatifloxacin and moxifloxacin are two fluoroquinolones in the third phase of clinical development. Phase I: safety, tolerability and pharmacokinetic assessment; phase II: safety and efficacy assessment on a small group of patients; phase III: randomised, controlled, multicentre study on a large number of patients to determine efficacy of a new drug relative to the gold standard regimen. LTBI: latent tuberculosis infection. Adapted from [43], with permission from the publisher.

quinolones. They further note concern for the measured high use of FQs given that recommendations and guidelines highlight the need for prudent FQ use and that these are not recommended as first-line treatment. This would be an indication of poor adherence to guidelines and recommendations. The study concludes by underlining that the excessive and inappropriate use of quinolones is not only associated with the development of resistance, but also higher costs for health providers and additional risk of adverse events for patients $[21,22,45]$.

Our survey showed that around half of current LRTI and CAP guidelines express the need for prudent FQ use, indicating them as the second drug of choice for treatment. We did not specifically look at the actual adherence to the guidelines or the actual use and prescription of FQs for these respiratory indications. However, the study by ADRIAENSSENS et al. [45] indicates that the use of FQs for outpatient care of lower respiratory infections is higher than warranted, indicating suboptimal adherence to guidelines.

\section{Conclusion}

The meta-analysis performed to assess whether treatment with FQs before the diagnosis of TB is associated with a higher risk of FQ-resistant TB suggests this is indeed the case. Whilst a number of national and sub-national guidelines on treatment of LRTI and/or CAP include the need to consider TB as a differential diagnosis and also the prudent use of FQs given the risk for FQ resistance, several guidelines are still lacking this consideration.
If the incidence of MDR-/XDR-TB, as well as TB resistant to all drugs, is to be curbed and decreased through enhanced control practices, it is essential to assure the rational use of drugs, not only for TB but also in other LRTIs. As countries reach the elimination phase of $\mathrm{TB}$, the knowledge among healthcare workers will also change and, thus, the challenge lies in maintaining the know-how and awareness of TB, its diagnosis and its treatment. Therefore, reaching healthcare workers through other healthcare system channels becomes an important action as TB care becomes decentralised and national TB programmes become horizontal rather than vertical.

The measured increased risk for FQ resistance upon exposure to FQs in potentially misdiagnosed TB patients, as shown in this systematic review, highlights the urgency to further strengthen and adhere to national and sub-national guidelines to ensure this trend is interrupted.

The international community has just taken a breath after agreeing on the XDR-TB definition in 2006 and discussions have been initiated on how to define the most advanced levels of drug resistance [12-14]. Agreement on these definitions is complicated by the different panels of second-line drugs tested in different countries and laboratories and by the new drugs recently used to treat these difficult cases (e.g. linezolid) [46]. FQs are key drugs in the treatment of TB; FQ resistance is a XDR-TB defining marker and, even in MDR-TB cases which are not yet XDR, it leads to worse prognosis (failure and death) [20]. In light of new anti-TB drugs predicted to become available within the next 2 yrs, the need to reinforce advocacy and training on the rational use of antibiotics is evident. 


\section{SUPPORT STATEMENT}

Part of this study (systematic review and meta-analysis) was financially supported by a European Centre for Disease Prevention and Control direct service contract (publication reference OJ/2010/07/ 12-PROC/2010/034). G.B. Migliori, L. D'Ambrosio and R. Centis are supported by a contribution of the European Commission through the FP7 PAN-NET project.

\section{STATEMENT OF INTEREST}

None declared.

\section{ACKNOWLEDGEMENTS}

The authors wish to thank the following for responding to the survey questionnaire and the ERS (Lausanne, Switzerland) for supporting the process: H. Flick and S. Hartl (Austria); R. Louis (Belgium); Y. Ivanov (Bulgaria); J. Skricková (Czech Republic); T. Ringbaek (Denmark); K. Kliiman (Estonia); T. Vasankari (Finland); T. Chinet (France); B. Schaaf and G. Höffken (Germany); E. Tzortzaki (Greece); E. Moloney (Ireland); F. de Blasio (Italy); R. Snipe (Latvia); E. Danila (Lithuania); R. Muller (Luxembourg); C. Farrugia Jones (Malta); P. Sterk (the Netherlands); K. Ronning (Norway); J. Domagala-Kulawik (Poland); J. Cardoso (Portugal); D. Bumbacea (Romania); I. Solovic (Slovakia); E. Quintana (Spain); and I. Abubakar (UK).

We would like to thank R. Spijker (medical information specialist; Academic Medical Center Amsterdam, Amsterdam, the Netherlands) for performing the search strategy and S. Hoffner (director of the WHO Supranational TB Reference Laboratory; Swedish Institute for Communicable Disease Control, Stockholm, Sweden) for his advice on the methods used for measuring FQ-resistance. We also thank L. Hooft (senior epidemiologist; Dutch Cochrane Centre, Amsterdam, the Netherlands) for critically reading the manuscript.

\section{REFERENCES}

1 Raviglione MC, Lange C, Migliori GB. Preventing and managing antimicrobial resistance: an imperative for chest physicians. Eur Respir J 2011; 37: 978-981.

2 Leung E, Weil DE, Raviglione M, et al. The WHO policy package to combat antimicrobial resistance. Bull World Health Organ 2011; 89: 390-392.

3 Zumla A, Blasi F, Raviglione MC. Rational use of anti-tuberculosis drugs in the EU: better patient care and less drug resistance. Eur Respir J 2012; 39: 802-804.

4 World Health Organization. Global Tuberculosis Control 2011. WHO/HTM/TB/2011.16. Geneva, World Health Organization, 2011.

5 Sotgiu G, Lange C, Centis R, et al. Extensively drug-resistant tuberculosis: back to the future. Eur Respir J 2010; 36: 475-477.

6 Veen J, Migliori GB, Raviglione M, et al. Harmonisation of TB control in the WHO European region: the history of the Wolfheze Workshops. Eur Respir J 2011; 37: 950-959.

7 Broekmans JF, Migliori GB, Rieder HL, et al. European framework for tuberculosis control and elimination in countries with a low incidence. Recommendations of the World Health Organization (WHO), International Union Against Tuberculosis and Lung Disease (IUATLD) and Royal Netherlands Tuberculosis Association (KNCV) Working Group. Eur Respir J 2002; 19: 765-775.

8 Migliori GB, Sotgiu G, D'Ambrosio L, et al. TB and MDR/XDR-TB in European Union and European Economic Area countries: managed or mismanaged? Eur Respir J 2012; 39: 619-625.

9 Skrahina A, Hurevich H, Zalutskaya A, et al. Alarming levels of drug-resistant tuberculosis in Belarus: results of a survey in Minsk. Eur Respir J 2012; 39: 1425-1431.

10 Sotgiu G, Ferrara G, Matteelli A, et al. Epidemiology and clinical management of XDR-TB: a systematic review by TBNET. Eur Respir J 2009; 33: 871-881.
11 Migliori GB, Besozzi G, Girardi E, et al. Clinical and operational value of the extensively drug-resistant tuberculosis definition. Eur Respir J 2007; 30: 623-626.

12 Migliori GB, Centis R, D'Ambrosio L, et al. Totally drug-resistant and extremely drug-resistant tuberculosis: the same disease? Clin Infect Dis 2012; 54: 1379-1380.

13 Migliori GB, Loddenkemper R, Blasi F, et al. 125 years after Robert Koch's discovery of the tubercle bacillus: the new XDR-TB threat. Is "science" enough to tackle the epidemic? Eur Respir J 2007; 29: $423-427$.

14 Migliori GB, De Iaco G, Besozzi G, et al. First tuberculosis cases in Italy resistant to all tested drugs. Euro Surveil 2007; 12: E070517.1.

15 Caminero JA. Likelihood of generating MDR-TB and XDR-TB under adequate National Tuberculosis Control Programme implementation. Int J Tuberc Lung Dis 2008; 12: 869-877.

16 Mitchison DA. The diagnosis and therapy of tuberculosis during the past 100 years. Am J Respir Crit Care Med 2005; 171: 699-706.

17 Falzon D, Jaramillo E, Schünemann HJ, et al. WHO guidelines for the programmatic management of drug-resistant tuberculosis: 2011 update. Eur Respir J 2011; 38: 516-528.

18 Migliori GB, Zellweger JP, Abubakar I, et al. European Union standards for tuberculosis care. Eur Respir J 2012; 39: 807-819.

19 Migliori GB, Sotgiu G, Blasi F, et al. Towards the development of EU/EEA Standards for Tuberculosis Care (ESTC). Eur Respir J 2011; 38: 493-495.

20 Migliori GB, Lange C, Girardi E, et al. Fluoroquinolones: are they essential to treat multidrug-resistant tuberculosis? Eur Resp J 2008; 31: 904-910.

21 Woodhead M, Blasi F, Ewig S, et al. Guidelines for the management of adult lower respiratory tract infections. Eur Respir J 2005; 26: $1138-1180$

22 Woodhead M, Blasi F, Ewig S, et al. Guidelines for the management of adult lower respiratory tract infections - full version. Clin Microbiol Infect 2011; 17: Suppl. 6, E1-E59.

23 European Centre for Disease Prevention and Control/WHO Regional Office for Europe. Tuberculosis surveillance in Europe 2009. Stockholm, European Centre for Disease Prevention and Control, 2011.

24 European Centre for Disease Prevention and Control. Progressing towards TB elimination. Stockholm, European Centre for Disease Prevention and Control, 2010.

25 Tuberculosis Coalition for Technical Assistance. International Standards for Tuberculosis Care (ISTC), 2nd Edn. The Hague, Tuberculosis Coalition for Technical Assistance, 2009.

26 Isaakidis P, Cox GS, Varghese B, et al. Ambulatory multi-drug resistant tuberculosis treatment outcomes in a cohort of HIVinfected patients in a slum setting in Mumbai, India. PlosOne 2011; 6: e28066.

27 Sandgren A, Cuevas LE, Dara M, et al. Childhood tuberculosis: progress requires advocacy strategy now. Eur Respir J 2012 [Epub ahead of print DOI 10.1183/09031936.00187711]

28 World Health Organization, Stop TB Partnership. The Global Plan to Stop TB 2011-2015. Transforming the Fight Towards Elimination of Tuberculosis. WHO/HTM/STB/2010.2. Geneva, World Health Organization, 2010.

29 van den Boom M, Seita A, Ottmani S, et al. Finding the way through the respiratory symptoms jungle: PAL can help. Eur Respir J 2010; 36: 979-982.

30 Chen TC, Lu PL, Lin CY, et al. Fluoroquinolones are associated with delayed treatment and resistance in tuberculosis: a systemic review and meta-analysis. Int I Infect Dis 2011; 15: e211-e216.

31 Shea BJ, Grimshaw JM, Wells GA, et al. Development of AMSTAR: a measurement tool to assess the methodological quality of systematic reviews. BMC Med Res Methodol 2007; 7: 10.

32 CHINAL. www3.cinahl.com/home.php Date last accessed: December 2010. 
33 TRIP database. www.tripdatabase.com/ Date last accessed: December 2010.

34 World Health Organization. International Clinical Trials Registry Platform. www.who.int/ictrp/en/ Date last accessed: December 2010.

35 Wells GA, Shea BJ, O'Connell D, et al. The Newcastle Ottawa Scale (NOS) for assessing the quality of nonrandomized studies in metaanalyses. www.ohri.ca/programs/clinical_epidemiology \oxford.htm Date last accessed: December 2010.

36 Jeon CY, Calver AD, Victor TC, et al. Use of fluoroquinolone antibiotics leads to treatment delay in a South African gold mining community. Int J Tuberc Lung Dis 2011; 15: 77-83.

37 Ginsburg AS, Hooper N, Parrish N, et al. Fluoroquinolone resistance in patients with newly diagnosed tuberculosis. Clin Infect Dis 2003; 37: 1448-1451.

38 Park I-N, Hong S-B, Oh Y-M, et al. Impact of short-term exposure to fluoroquinolones on ofloxacin resistance in HIV-negative patients with tuberculosis. Int J Tuberc Lung Dis 2007; 11: 319-324.

39 Wang J-Y, Lee L-N, Lai H-C, et al. Fluoroquinolone resistance in Mycobacterium tuberculosis isolates: associated genetic mutations and relationship to antimicrobial exposure. J Antimicrobial Chemotherapy 2007; 59: 860-865.
40 Long R, Chong H, Hoeppner V, et al. Empirical treatment of community-acquired pneumonia and the development of fluoroquinolone-resistant tuberculosis. Clin Infect Dis 2009; 48: 1354-1360.

41 Devasia RA, Blackman A, Gebretsadik T, et al. Fluoroquinolone resistance in Mycobacterium tuberculosis. The effect duration and timing of fluoroquinolone exposure. Am J Crit Care Med 2009; 180: 365-370.

42 Ma Z, Lienhardt C, McIlleron $\mathrm{H}$, et al. Global tuberculosis drug development pipeline: the need and the reality. Lancet 2010; 375: 2100-2109.

43 Stop TB Partnership. Working Group on New TB Drugs. Drug pipeline. www.newtbdrugs.org/pipeline.php Date last accessed: January 2012.

44 Caminero JA. Multidrug-resistant tuberculosis: epidemiology, risk factors and case finding. Int J Tuberc Lung Dis 2010; 14: 382-390.

45 Adriaenssens N, Coenen S, Versporten A, et al. European Surveillance of Antimicrobial Consumption (ESAC): outpatient quinolone use in Europe (1997-2009). J Antimicrob Chemother 2011; 66: Suppl. 6, vi47-vi56.

46 Villar M, Sotgiu G, D'Ambrosio L, et al. Linezolid safety, tolerability and efficacy to treat multidrug- and extensively drug-resistant tuberculosis. Eur Respir J 2011; 38: 730-733. 